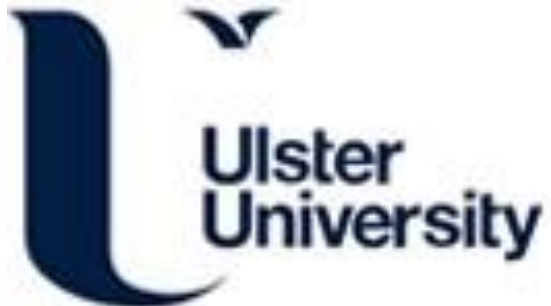

\section{Motion-Onset Visual Evoked Potentials for Gaming}

Marshall, D., Wilson, S., \& Coyle, D. (2015). Motion-Onset Visual Evoked Potentials for Gaming. In Unknown Host Publication (pp. 155-164). Global Science and Technology Forum. https://doi.org/10.5176/22511679_CGAT15.41

Link to publication record in Ulster University Research Portal

\section{Published in:}

Unknown Host Publication

Publication Status:

Published (in print/issue): 01/01/2015

DOI:

10.5176/2251-1679_CGAT15.41

\section{Document Version}

Publisher's PDF, also known as Version of record

\section{General rights}

Copyright for the publications made accessible via Ulster University's Research Portal is retained by the author(s) and / or other copyright owners and it is a condition of accessing these publications that users recognise and abide by the legal requirements associated with these rights.

\section{Take down policy}

The Research Portal is Ulster University's institutional repository that provides access to Ulster's research outputs. Every effort has been made to ensure that content in the Research Portal does not infringe any person's rights, or applicable UK laws. If you discover content in the Research Portal that you believe breaches copyright or violates any law, please contact pure-support@ulster.ac.uk. 


\title{
Motion-Onset Visual Evoked Potentials for Gaming
}

\author{
Mr David Marshall, Dr Shane Wilson, Dr Damien Coyle, \\ School of Computing and Intelligent Systems, University of Ulster Magee \\ University of Ulster
}

\begin{abstract}
Motion onset visually evoked potentials (mVEPs) allow users to interact with technology using non-visually fatiguing stimuli in a brain-computer interface (BCI). This study employs mVEP for brain controlled computer games and evaluates players ability to use mVEP for online gameplay with games from three different genres namely action, puzzle and sports. Five mVEP stimuli are presented as buttons to allow the subject to choose from five possible actions in each game. The performance was assessed based on offline and online BCI accuracy and game score. The results indicate that the players could control the games with reasonable online accuracy $(65 \%$ average for 5 class classification, with an average training accuracy of $74 \%$ ). The study also found that players did noticeably better in the sports game (bowling) than other game genres in both online BCI testing and game playing scores.
\end{abstract}

Keywords: Brian Computer Interface, Gaming, Game Genre, Control Methods

\section{INTRODUCTION}

Many people with motor impairments cannot use conventional control devices such as a mouse, keyboard or game controller. Individuals with no motor control cannot rely on interfaces such as mouth sticks; eye tracking or electromyogram (EMG) switches (a switch that can convert electronic signals on the skin into signals that can be used in assistive equipment). Brain-computer interfaces (BCIs) have the potential to enable these users to control and interact with devices and technology using directly measured brain activity [1][2][3]. An electroencephalography EEG-based BCI measures voltage fluctuations resulting from ionic current flows within the neurons of the brain via electrodes placed upon the scalp, translating these signals into commands for a program to execute [1]. Recently there has also been interest in the application of BCI's for able-bodied users across a number of application domains such as the automotive and entertainment industries [5] [6] [7].

In recent years the application of BCI for interacting with computer games has become increasingly popular across many BCI research studies. BCI games are often used to test paradigms or train users how to use $\mathrm{BCI}$ and have become increasingly more advanced; utilizing 3D environments, multiple user objectives and hybrid control systems which incorporate both conventional input devices and multiple BCI techniques [8] [9].

Visual evoked potentials (VEPs) have frequently been used in BCI systems, with visual stimuli such as flashing lights (P300) or pattern reversal (SSVEP) being utilized to elicit signals from the primary visual cortex. Recently however BCI studies [10] [11] have focused upon VEPs that do not incorporate such alternating stimuli. Motion-onset visual evoked potentials (mVEP) is a promising paradigm for VEP BCI due to its large amplitude, low inter- and intra-subject variability and the use of elegant and simplistic stimuli. This paper focuses on assessing mVEP-BCI as a control methodology in a range of computer game genres and identifying the most appropriate genre or genres for the paradigms use.

Many research studies have begun to focus upon the entertainment elements of $\mathrm{BCI}$ games and gaming. In recent years the application of BCI for interacting with computer games has become increasingly popular amongst many BCI research studies with a large upsurge of studies within the last 7 years [9][12]. The research and technical challenges associated with the development of BCI games vary considerably depending on the type of BCI paradigm being employed as well as the genre and inherent gameplay challenges.

Motor imagery involves the imagination of the movement of various body parts resulting in sensorimotor cortex activation which modulates sensorimotor oscillations in the EEG. This can be detected by the BCI to infer a user's intent. Motor imagery typically requires a number of sessions of training before acceptable control of the BCI is acquired. These training sessions may take a number of hours over several days before users can consistently employ the technique with acceptable levels of precision. Lengthy training sessions are uncommon even within the most complex of games. Instead designers incorporate tutorials within the gameplay goals of early levels gradually introducing more complex interaction schemes and gameplay objectives as the game progresses. Motor imagery is typically suited to action games as it allows a continuous control method that can be used in combination with simple gameplay challenges $[13][14][15][16]$.

Passive BCI involves using BCI to enrich human-machine interaction with implicit information on the actual user's state, for example simulations to detect when a user is losing concentration when driving [17]. There are many examples of passive BCI usage in games [18] [19] [20]. Alphawow [21] runs on a modified version of the popular massively multiplayer game World of Warcraft, one aspect of the characters actions is controlled via passive BCI, the characters form. The character within Alphawow has two forms, an elf and a bear. The elf form allows them to attack enemies from a safe range and the bear allows them to attack from close range (the bear is also more resistant to attacks). The "shape shifting" in the game is controlled via the user's parietal alpha activity. This means that when the player is relaxed they will 
remain in elf form (the player is safe and attacking from a distance) and when the player becomes agitated they will transform to bear form (the player is under attack and needs to defend himself/herself or attack the enemy). Passive BCI can be used in all genres of games but generally will only control one element while the player controls using BCI or a traditional control method.

Visual evoked potentials have also been used extensively in BCI games. A VEP (visual evoked potential) is an electrical potential recorded after a subject is presented with a type of visual stimulus. There are several types of VEPs. Steady-state visually evoked potentials (SSVEPs) use potentials generated by exciting the retina using visual stimuli modulated at certain frequencies. SSVEPs stimuli are often formed from alternating checkerboard patterns [22] and at times simply use flashing images [23] [24]. Another type of VEP used with applications is the P300 evoked potential. The P300 event-related potential is a positive peak in the EEG that occurs at roughly $300 \mathrm{~ms}$ after the appearance of a target stimulus (a stimulus for which the user is attending or seeking) or oddball stimuli [24].

Visual evoked potentials have been used in a wide range of game genres. For example a Simple action games to control a characters balance. In "MindBalance" [25] a player must balance an onscreen avatar across a tightrope using SSVEP symbols. P300 event-related potentials have also been widely used in VEP BCI's. For example in the "Mindgame" [26], a player cuts down trees within a 3D game board. The player's task is to strategically control the avatars path from tree to tree. The player must choose the quickest or shortest route between the trees as the players "confidence" or the measure of the quality of the P300 affects the number of steps the avatar takes between trees.

As flash or pattern reversal VEP based BCIs use a high contrast or bright luminance of visual stimuli they can cause notable visual fatigue on the BCI user. It is therefore important to consider these factors given knowledge about the end use of BCI as many of these VEPs depend upon environments without poor target contrast or fluctuant luminance such as a user's home or a clinical bedside. In contrast mVEP is elicited entirely by the motion behavior of the visual object and is not sensitive to the contrast and the luminance of the object or the area around it [10].

MVEP incorporates neural activity from the dorsal pathway of the visual system which allows more elegant visual stimuli than the aforementioned types of VEP (P300 and SSVEP) [10]. Among all visual motion related VEPs mVEP displays the largest amplitudes and the lowest inter- and intra-subject variability rendering it suitable for use within a BCI application. Motion-onset VEP is typically composed of three main peaks: P1, N2 and P2. The negative N2 peak, with a latency of $160-200 \mathrm{~ms}$, is motion specific. The positive P2 peak has a latency of around $240 \mathrm{~ms}$ and is increased with more complex visual stimuli. These clear and robust temporal features make mVEP a promising EEG component for information encoding and decoding within a BCI system.

The first notable use of mVEP was within a simple testing BCI environment [10] where a virtual keyboard was used to enable the recording of data from a subject in both offline and online testing. The subject gazed at the desired onscreen button (an mVEP symbol); the brief motion of the symbol (a bar moving from left to right (Fig. 1)) elicited the mVEP. The EEG data segment taken was aligned to the motion onset of the chosen target and contained prominent motion related VEP features. The spatio-temporal pattern of mVEP in this paradigm was investigated by using EEG data from 15 subjects. N2 and P2 components of mVEP from temporooccipital and parietal electrodes are selected as salient markers of brain responses to the attended target. By averaging aligned mVEP signals from multiple trials for each moving object, the time-locked response of the attended target was enhanced. The stimulus producing the largest N2/P2 component was identified as the intended target. Besides a simple feature extraction of N2/P2 area calculation, the widely used stepwise linear discriminant analysis (SWLDA) in a P300 speller was adopted to assess the target detection accuracy of a five-class mVEP BCI. Within this trial a mean of $98 \%$ accuracy was achieved when averaging over 10 trials using 15 subjects [10]. mVEP has also been used within n200 spelling applications[11]. The n200 speller uses the same rectangular symbols used within [10], however in this study the symbols were incorporated within a matrix of 36 virtual onscreen buttons (much like the P300 speller). The user was required to focus their attention toward the button labelled with the letter to be communicated. The computer then determined the target letter by identifying the attending row and column respectively. Ten users had a mean accuracy of $91 \%$ using a single channel and an average of 4.1 trials compared to the P300 speller using a single channel which achieved a mean accuracy of $72 \%$.

Before conducting a study into mVEP's effectiveness as a control scheme in computer games a pilot study was conducted to gauge its success when using the proposed system [27] (BCI system and a commercial games engine). The pilot study allowed adjustments to the paradigm, system and game engine architecture before testing the games with a larger group of participants. This study was conducted to determine the most appropriate methods, parameters and EEG setup to use in order to extract reliable information when classifying responses on up to five different stimuli in a simple environment within a game engine. Initial offline results showed that $80 \%$ accuracy can be achieved by averaging stimuli over 5 trials i.e., 5 seconds of data, when discriminating target versus non target. This was achieved by the use of simple averaging techniques and support vector machines. 
As it has been established that mVEP represents an appropriate VEP for use as a control signal within a BCI-game framework. The objectives of this study are to create BCI games that test the mVEP paradigm in several computer game environments where the user interacts with the games directly using the BCI system. This study uniquely uses the BCI paradigm in three different game genres so to find the most appropriate genre and gameplay method for this paradigm.

\section{METHODS}

\section{A. System}

The mVEP BCI system comprises of several different components both physical and software based. The commercial game engine Unity 3D [28] was used to develop the games and present all the visual stimuli to the user. As Unity $3 \mathrm{D}$ renders the visual stimuli to the screen, data packets describing these stimuli events are transmitted to Matlab [29] over a User Datagram Protocol (UDP). UDP was selected as the communication protocol as it allowed Unity 3D to transmit messages without requiring special transmission channels or data paths. Upon receiving a UDP packet the Matlab component processes the game event data and user EEG signals in real-time.

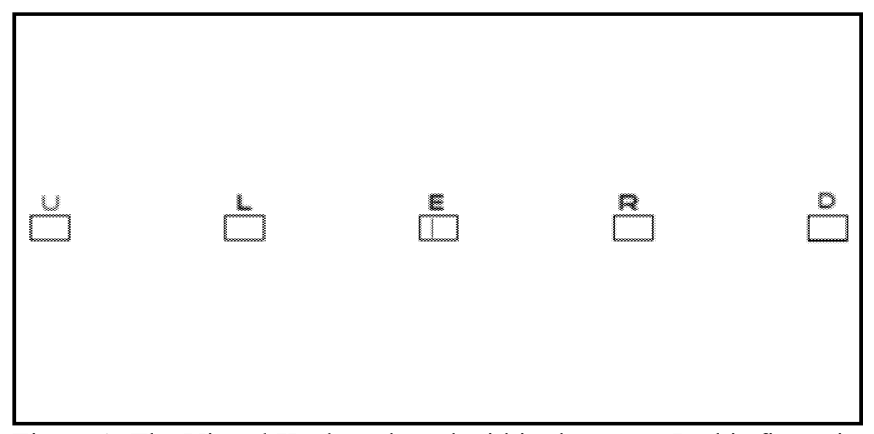

Figure 1: The Virtual Keyboard used within the game. In this figure it shows the virtual keyboard with the target letter $U$ highlighted. This tells the participant to gaze at the symbol below $U$. E is active in this example with the red horizontal line in the symbol moving from right to left; the participant will ignore this symbol movement. Here the $\mathrm{U}$,

$\mathrm{L}, \mathrm{E}, \mathrm{R}$, and $\mathrm{D}$ are a reference to up, down, enter, left, and right.

\section{B. Training}

Visual stimuli were displayed on a white board within the game environment and viewed on a 22 inch LCD monitor within a $60 \mathrm{~Hz}$ refresh rate. Each symbol is a small rectangle of $1.24^{\circ}$ by $0.76^{\circ}$ visual angle. The rectangles when active contain a red vertical line with a $0.66^{\circ}$ visual angle appearing in the right side of the vacant rectangle and is moved leftward at a velocity of $3.10^{\circ}$ before it disappears (this process of motion took $140 \mathrm{~ms}$ ) e.g., see symbol E in Fig. 1.

Each symbol had a letter placed above it, the letter would dictate what symbol the user is required to look at. When the letter is highlighted red the user must focus on the symbol. These symbols or buttons form a virtual games controller $\mathrm{U} / \mathrm{L} / \mathrm{E} / \mathrm{R} / \mathrm{D}$ (originally used for directional controls, but changed for this control method).
The timing scheme of the stimuli followed the scheme presented in [10] yet was significantly shorter with a single block consisting of 5 trials taking 8 seconds as opposed to 15 trials in a block taking 24 seconds [10], when a block is complete each symbol within the block will have moved 5 times (one for each trial). In a trial each symbol is activated once, this is randomly designated with no overlap. The stimulus onset asynchrony (SOA) between two motion stimuli is $200 \mathrm{~ms}$.

For training, in each block the subject is asked to focus on one symbol (letter highlighted). Each trial consists of each symbol moving for a period of $140 \mathrm{~ms}$ then a static phase of $60 \mathrm{~ms}$, after which the next randomly selected stimuli is initiated. This is repeated until all 5 symbols have completed their animation (therefore lasting 1,000ms) Fig. 2 shows the proposed timing scheme. For offline training each run lasts approximately 10 minutes and consists of 60 blocks with each block containing 5 trials each. Using the data collected in training the system is able to find the correct parameter data for use in the three games.

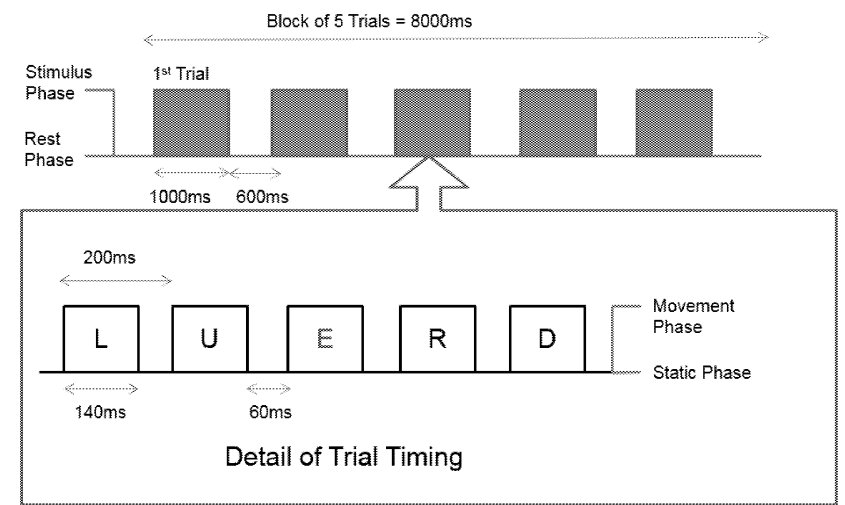

Figure 2: The timing protocol of one data acquisition period (one block): each block consists of 5 trials. Each trial is subdivided into five stimulus periods dedicated to the five virtual buttons respectively. SOA (stimulus-onset asynchrony) was $200 \mathrm{~ms}$. The motion stimuli indicating the five buttons appear in random order, with one button ( $\mathrm{E}$ in this case) designated as the target. This is based on the timing paradigm proposed in [10].

The training level run twice. This was done to determine how the classifier trained on training run 1 performed on training run 2. This was done to compare the performance of the training paradigm which has no feedback and white background compared to the games where real-time feedback is provide and the background and graphics differ for each game on the performance on the training paradigm Results were analysed to determine if performance was degraded or improved by feedback. This online testing using the training paradigm is named "Training Paradigm Test".

\section{Games}

The testing of each game consisted of two stages. The first stage consisted of the player's choices being directed by the game (by an arrow above the selected symbol), this stage 
lasted approximately 10 minutes. This stage allowed to gauge the accuracy of the online system and player while playing the games. Within the second stage of the games the player was asked to simply play the game using their basic knowledge of the games mechanics to achieve the highest score possible. Each game had 8 control stages allowing each genres scores to be compared. Within each game during the control period the symbols are placed and sized exactly the same as within the training phase. The order of the games was shuffled randomly to prevent confounding results based on order of games played.

\section{1) Action game}

An action game was created allowing the player to move through a detailed virtual environment automatically whilst performing tasks and interacting with the games using the mVEP buttons. The game environment included graphical aspects found in most modern games such as dynamic shadows, reflections and animated characters (Fig. 3). Using a detailed virtual environment the mVEP paradigm's application in more detailed games with moving distractions can be tested. The sections of the action game that allowed for player control are hostage situations with the player's objective being to target the hostage taker and free the captives. The player is presented with five different options with only one option being correct. The correct option is always very clear with the hostage taker being the only individual with a weapon.

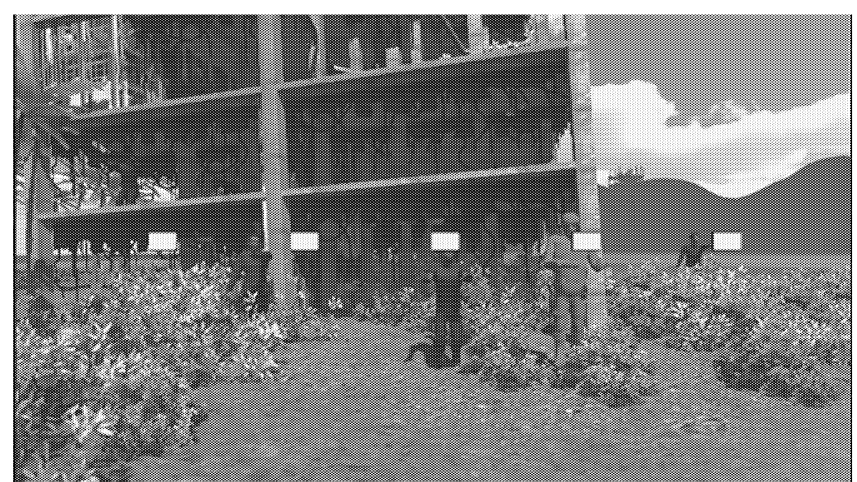

Figure 3: The player is presented with a detailed world with moving characters and is required to choose what character to shoot. The stimuli are placed in exactly the same area as within training.

As the game is an action game the player is given a short time (three seconds) to decide what character to target. After the three seconds the player must then concentrate on the symbol in front of the chosen character. The mVEP stimuli will then begin to cycle with the player concentrating on the required symbol. After this the BCI signal processing framework in Matlab triggers and processes the data returning the classification result via UDP to the game detailing the selected stimulus. The character behind that stimulus will then collapse and the player is given a point if the correct character was selected. The player's character is then automatically transitioned through the game environment to the next stage of the game. The game consists of 8 different stages each becoming slightly more complex, in some stages the player's camera must firstly zoom to show the positions of the characters and in other stages the characters may be slightly obscured by objects in the game environment. Even with a slight difficulty increase throughout the game it is still obvious which target the player should choose throughout.

\section{2) Puzzle game}

A simple puzzle game was created to allow testing of the puzzle genre. The puzzle genre was deemed most appropriate for use of VEP's within [9]. The puzzle game is a simple physics game with a ball being dropped from five tubes and required to pass by objects to reach a collection zone at the bottom of the level (Fig. 4). This game emulates games such as the physical "coin drop" games found in arcades.

The player is given a longer time to decide what stimuli to concentrate on (ten seconds). This allows the player to approximate the balls path through the obstacles from each drop point. The symbols are located at the top for the player to choose the initial drop point and the basket placed at the bottom in a position fixed at the beginning of that round. After ten seconds the camera zooms into the symbols so they are an appropriate size and separation for mVEP control and the player will choose the selected drop point. After the symbols have cycled and the system has identified the selected symbol the camera zooms out and the ball is dropped from the appropriate drop point. If the player's ball enters the collection point at the bottom of the level the player will be awarded a point and move onto the next stage.

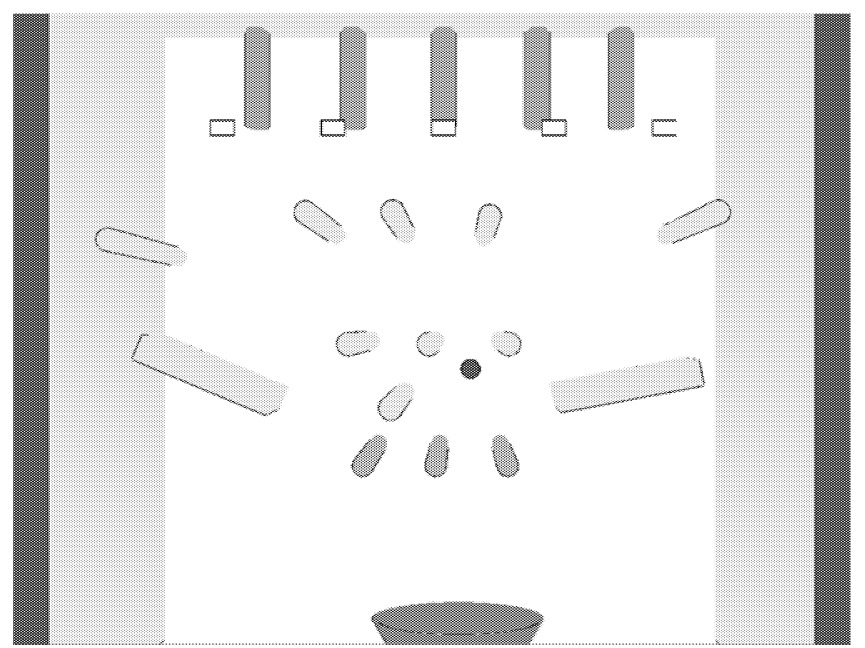

Figure 4: The second level of the puzzle game with the drop points located at the top beside the appropriate symbols and the green collection point placed at the bottom of the level. The player must choose the correct drop point to fall (the camera repositions so to resize the symbols to the required visual angles) and bounce past the beige tubes to reach the collection point

The puzzle game consists of eight stages each getting slightly more difficult with more obstacles of different shapes being added and the collection points position being changed. The changing of the game environment allows for an elegant 
difficulty curve with the player becoming more accustomed with how the ball reacts to obstacles as the game progresses.

\section{3) Sport game}

A sports game based around ten pin bowling was created. This game allows the player to participate in two rounds of bowling with control over the balls bowling position and direction. The player is presented with a typical bowling alley with ten bowling pins to knock over within two shots. The player must firstly choose the balls position and then from that position choose to put a spin on the ball so that it will curve in the spin direction (Fig. 5).

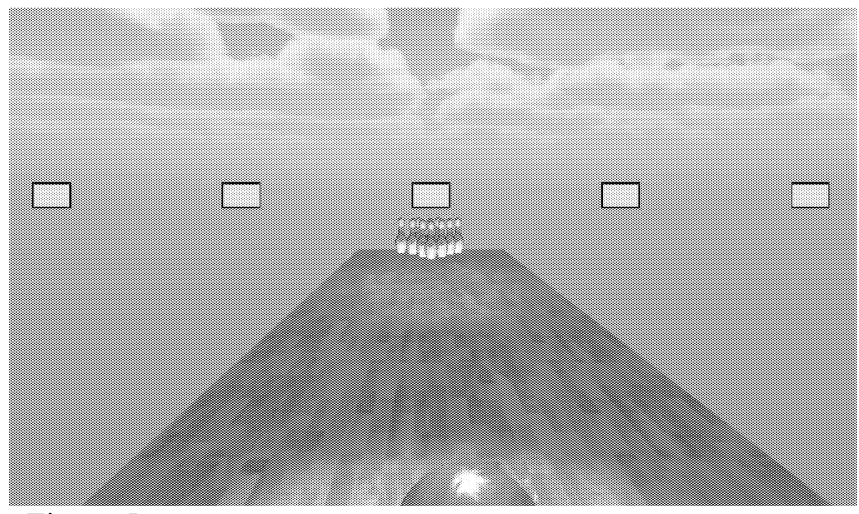

Figure 5: The bowling alley setup. The symbols are located above each of the bowling starting points. In this screenshot the player is about to begin choosing their spin direction.

The sports game required two decisions, the decision time allowed was 5 seconds for each decision. The player is firstly shown the pins from a top view allowing them to better judge where to aim. The player then decides which one of five positions to bowl from, the mVEP symbols then begin to cycle. After the system has found the players intended bowling position the ball will drop into place. After that the player is allowed five seconds to decide what direction to spin the bowling ball, so to curve it. The symbols will then cycle again and the ball will be bowled. This allows the player to make corrections if the first decision period did not go as the player intended. After the pins have been knocked over the player will set up the second shot in the same way or begin a new round if they have knocked over all of the pins. The game consists of four rounds instead of eight as the player must make two mVEP decisions to cause an action in the game. The games difficulty remains balanced throughout this game with the player becoming more experienced as they play.

Using these games we aimed to identify what genre the player achieves the highest BCI accuracy in and also what genre the player achieves the highest score in. Using two performance metrics enables a more detailed analysis of the control methods usage in multiple game genres. We also compare performance with real-time game feedback and graphics display against real-time classification using the training paradigm with basic graphics (Games vs Training ParadigmTest).

\section{Data Acquisition}

One female and nine male subjects took part in this study. Three of these subjects had previous BCI experience using mVEP without feedback and the other subjects were BCI naive. Participants were seated on a comfortable chair and placed $50 \mathrm{~cm}$ (appropriate distance to maintain visual angles) in front of a 22 inch LCD monitor. Training and testing involved a single two hour session in which all games where tested using a single eight minute training session. During setup the EEG cap was placed over occipital areas using a 12 channel montage (Fig. 6) according to the international 10-20 system of electrode placement [30]. This montage placement was influenced by the results of an earlier pilot study [27]. The electrodes cover the optimal area for classifying mVEPs. The left mastoid was used as a ground and $\mathrm{FPz}$ as the reference.

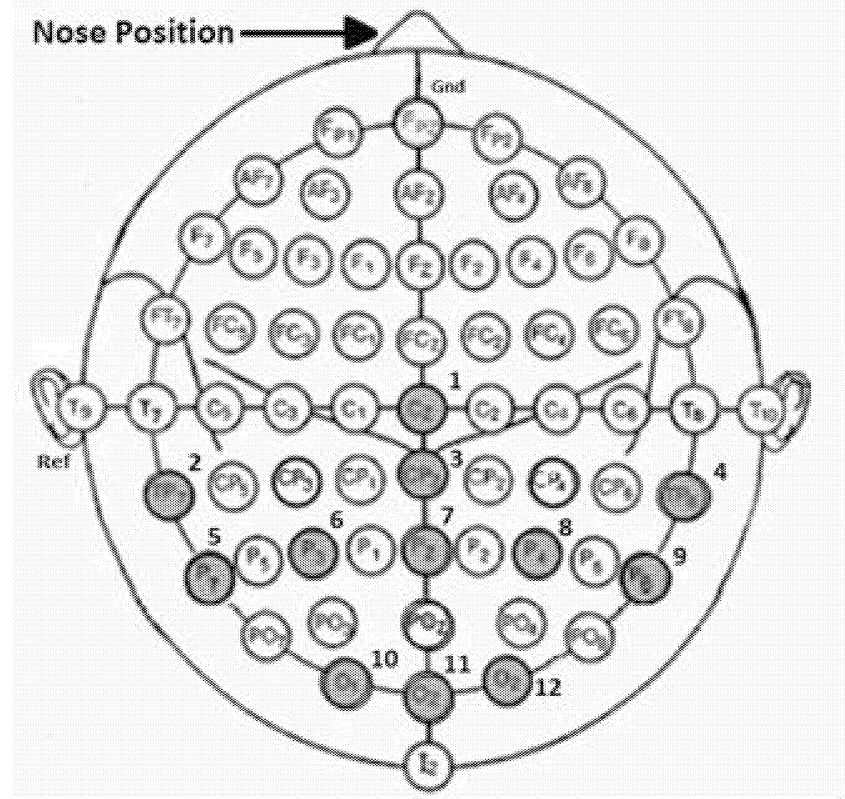

Figure 6: The electrode montage used with electrode show in grey

EEG data was collected using a g.BSamp amplifier [32] and g.Gammasys active electrode system [33]. The data was over sampled at $250 \mathrm{~Hz}$, then average down sampled to $125 \mathrm{~Hz}$.

\section{E. Data preprocessing Methods}

A total of 60 blocks were recorded from each subject during the training period. Data epochs were derived in association with each motion-onset stimulus, beginning $200 \mathrm{~ms}$ prior to the motion onset and lasting for $1200 \mathrm{~ms}$. All single trials were baseline corrected with respect to the mean voltage over the $200 \mathrm{~ms}$ preceding motion onset. Data was digitally filtered using a low-pass Butterworth filter (order 5, with cut of at $10 \mathrm{~Hz}$ ) and subsequently resampled at $2600 \mathrm{~Hz}$. Features were extracted between $100 \mathrm{~ms}$ and $500 \mathrm{~ms}$ (the epoch that normally contains the most reactive mVEP components e.g., N200, P300, N400) post stimulus yielding nine features for each channel. Data was averaged over 5 trials yielding 12 feature vectors per stimulus. Since mVEP is time locked and phase 
locked to the motion-onset stimulus, mVEP induced from the motion stimuli could be obtained through the above simple processing procedure. Data was split into target vs non-target as well as individually classed for each target stimuli where for each non-target feature vector five randomly selected nontarget trials were used.

\section{F. Channel selection}

A linear discriminant analysis (LDA) classifier was trained to discriminate target vs non-target feature vectors extracted from single channels in a leave one-out cross validation (LOO) on $50 \%$ of the data (the remaining $50 \%$ was held out for final offline testing). For each of the twelve channels the average LOO classification accuracy (LOO-CA) was determined and channels were ranked by accuracy. The three top ranked channels were concatenated to form a new feature vector (27 features per vector) and a further LOO crossvalidation was performed. A single trial test of target vs nontarget is also applied on the training data (Target vs Non Target - SingleTrial).

\section{G. $\quad$ mVEP classification - 5 class}

Using all the training data (50\% of recorded training data) a new LDA classifier is produced to classify target vs non-target data. To classify individual symbols in a single trial test each feature vector associated with each stimulus in a trial is classified as either target or non-target. The LDA classifier produces a distance value, $D$, reflecting the distance from the hyperplane separating target and non-target features $(D>0$ for target and $D<0$ for non-target). The vector that produces the maximum distance value is selected as the classified stimulus (in some cases non-target data produce a $D>0$ however the value of $D$ is normally maximal among the five stimuli for target stimulus i.e., the stimulus on which the user is focused). Single trial results for discriminating the five classes' offline are reported for the training data and then the setup is applied on the remaining $50 \%$ of the data, unseen testing data to give offline testing results prior to online real-time performance evaluation. Offline analysis was performed using customized code and the Biosig and LIBSVM toolboxes [31] [34].

\section{H. Realtime game control and online feedback}

Online BCI control of games actions involves using the classifier setup on the training data and the best three selected channels (Fig. 7). The online system used a Matlab session based approach that allows for data to be collected and analyzed in real-time in parallel to the three Unity based games. In the session based interface online real-time triggering is performed. In each game the user waits until options become available, then the stimuli are presented 5 times for each button (over 5 trials), the session based interface waits until the triggers associated with these stimuli are received, averages over the 5 trials, features are extracted as described in section II.E, the trained classifier is applied and the stimulus (button or action) is determined based on the maximal distance, $D$, as outlined in section II.G. The selected button is communicated to the game via UDP and the realtime feedback is provided to the user and points adjusted based on performance e.g., in the puzzle game the ball drops, in the action games the target character falls, in the bowling game the bowl moves to strike the pins.

An illustration of the online process is shown in Fig. 7.

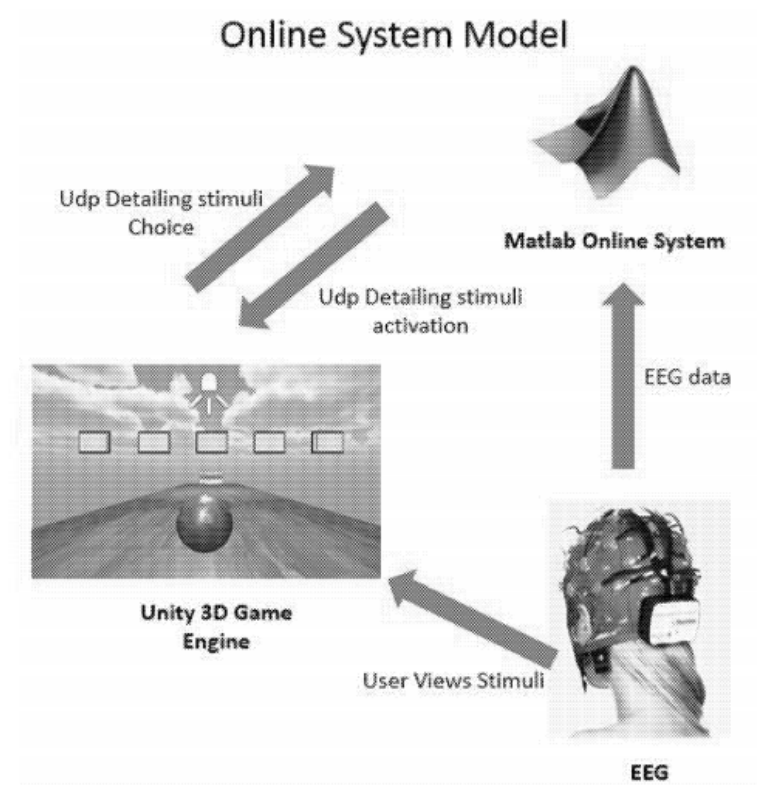

Figure 7: Online BCI system. Unity 3D displaying the stimuli and sending triggers in relation to the stimuli movement to the Matlab session based interface to co-register with the EEG. Signal processing is performed in Matlab to classify the EEG, returning back the label associated with the selected button.

\section{RESULTS}

Data recorded during both training and testing games was analyzed separately. Results are presented for training, training paradigm test (no feedback), the bowling game, the puzzle game and the action game.

\section{A. Offline Analysis}

Offline analysis was conducted over training and games. This allows for analysis of the system and recordings without the use of classification data recorded in training. Results are presented as LOO classification accuracy, Target Vs Non Target using training data, Single trial five class using training data and single trial five class testing offline(Table 1).

Table 1 shows the average LOO classification accuracy for the best 3 channels over the five runs. LOO classification accuracy did not vary significantly over the five runs with the action game producing $80.9 \%$ classification accuracy offline and the training producing $79.7 \%$. The use of LOO to find electrode placement found that the most common three electrodes are P7 01 and P3. These electrodes cover the area 
found in [10] to be best for leftward movement of the stimuli (the same stimuli used in this study). The asymmetrical topography of electrode selection may be explained by the right visual field asymmetry effect on contralateral hemisphere during selective attention [35]. It may also have bearing to the hemispheric asymmetry in human motion perception, as it was found that $\mathrm{N} 2$ is generated by extrastriate activity and that motion stimuli are not equivalently processed in the two cerebral hemispheres [36].

TABLE 1: This table shows the average over all subjects for all classification types. It is organized by what was assessed as training data, offline testing and Online testing with feedback. These are then categorized into Target vs Non Target LOO Classification and single trial five class Classification. Accuracies under training assessment used the training data (first 30 blocks) and accuracies under offline testing used the remaining data using classifiers gained from the first 30 blocks. Online testing accuracies used classifiers gained from the first $50 \%$ of the training offline data type (30 blocks).

\begin{tabular}{|c|c|c|c|c|}
\hline & \multicolumn{2}{|c|}{ Training Assessment } & \multirow{2}{*}{$\begin{array}{c}\text { Offline } \\
\text { Testing } \\
\text { Five } \\
\text { Class }\end{array}$} & \multirow{2}{*}{$\begin{array}{c}\text { Online } \\
\text { Testing } \\
\text { Five Class }\end{array}$} \\
\hline & $\begin{array}{c}\text { Target vs } \\
\text { Non } \\
\text { Target }\end{array}$ & Five Class & & \\
\hline Data Type & $\begin{array}{l}\text { LOO } \\
\text { Acc }\end{array}$ & $\begin{array}{l}\text { Single } \\
\text { Trial }\end{array}$ & $\begin{array}{l}\text { Single } \\
\text { Trial }\end{array}$ & $\begin{array}{l}\text { Single } \\
\text { Trial }\end{array}$ \\
\hline Training Offline & 79.667 & 91 & 82 & None \\
\hline $\begin{array}{l}\text { Training Paradigm } \\
\text { Test }\end{array}$ & 79.722 & 86.22222 & 71.44444 & 67.83 \\
\hline Bowling Guide & 80.194 & 92 & 75.55556 & 69.5 \\
\hline Puzzle Guide & 80.944 & 87.11111 & 73.66667 & 65.25 \\
\hline Shooting Guide & 79.444 & 86.22222 & 75.66667 & 60.25 \\
\hline Average & 80.076 & 87.889 & 74.083 & 65.707 \\
\hline
\end{tabular}

Fig. 8 shows the difference in the single trial 5 class accuracies when the classifier is trained on the first 30 blocks of data and applied on the first 30 blocks of data versus a classifier trained on the first 30 blocks of each data set and tested on the remaining data (Retrained Classifier), we called this "offline testing".

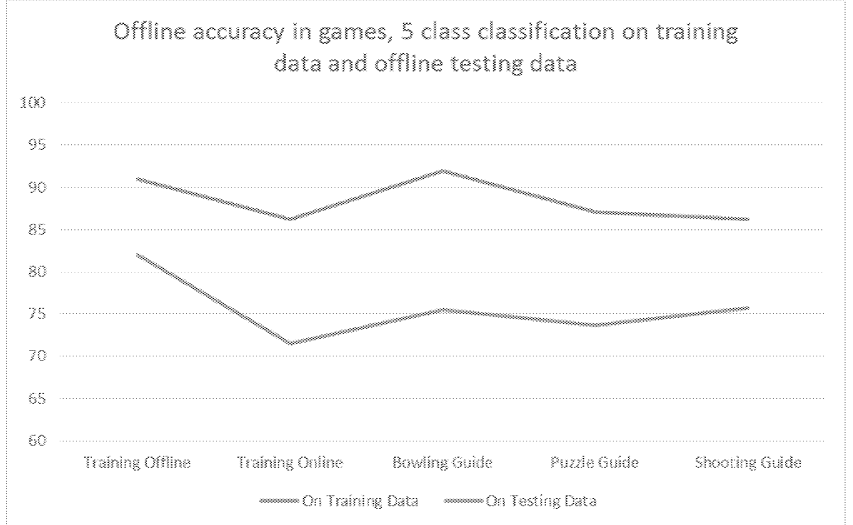

Figure 8: Classification accuracies on the training data (first 30 Blocks) in comparison to classification accuracies using offline testing data (remaining data).
Single trial 5 class accuracies using the training data are 10$15 \%$ higher than single trial 5 class testing offline accuracies, this is expected as they use the same training data as the data used to create the classifiers (first 30 blocks of data). Single trial five class accuracies using the training data allowed for high averages of $91 \% / 92 \%$ over all subjects in the training environment and bowling game. Single trial five class testing offline allowed for some subjects to achieve $100 \%$ accuracy yet in online five class tests these subjects could only achieve $95 \% / 90 \%$ accuracy. Using a single factor ANOVA on offline testing results it was found that difference between offline accuracies in games were not statistically significant $(p=0.914)$.

The training paradigm achieved a high single trial five class offline testing accuracy of $82 \%$ this suggests that if trained and then tested immediately afterwards most subjects can achieve high accuracies in a simple environment. For single trial five class offline testing using the games subjects achieved on average $74 \%$ this shows that the games do degrade performance yet subjects can still achieve good accuracies if trained within the games environments.

\section{B. Online Analysis}

Online Testing accuracies where measured by the recording of a subjects choices when controlling the game online. This used a classifier that was trained using the offline training data collected at the beginning of the session. The subject was guided by an arrow through the game and made 40 different selections through the online testing period. The subject was immediately given feedback within all games, whilst no feedback was given with the training paradigm test. In the games the subject was asked to concentrate on the stimuli with an arrow above it, the arrow would cycle above each stimuli in order and then reset (much like the red letters in the training paradigm). During this testing visual distractions within the game where active (moving characters, camera movements and responses from the players choices).

The BCI accuracy during online game testing percentage is measured by the percentage of correctly chosen stimuli (if the arrow is above symbol one, the subject is directed to choose symbol one). The results for each are shown in Fig. 9. On average subjects were able to achieve $65.7 \%$ online accuracy. Subjects attained highest accuracies in the training paradigm test $(67.8 \%)$ and bowling $(69.5 \%)$ game. The puzzle game produced $65.25 \%$ accuracy whilst the shooting game achieved the lowest accuracy $60.25 \%$ accuracy. Using a single factor ANOVA on online testing results it was found that difference between accuracies in games were not statistically significant $(p=0.537)$. 


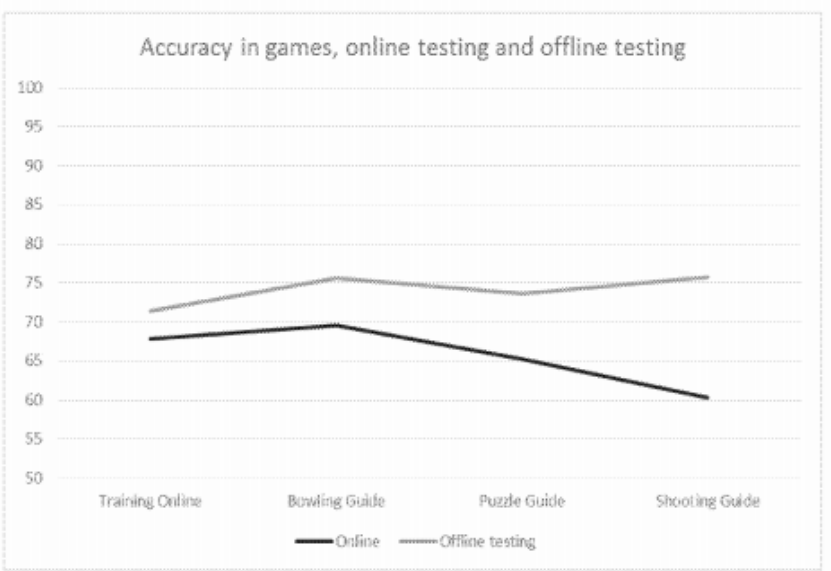

Figure 9: The average online accuracy for all subjects across all games in comparison to the offline testing averages.

In Fig. 9 and (Table 1) it shows that there is a clear $5 \%-15 \%$ difference in average offline testing (using a retained classifier from the first 30 blocks of data on the remaining data) and online testing results. As well as a slight downward trend in online testing accuracy over the three games.

Four of the subjects achieved above $70 \%$ online accuracy averaged over the 4 control stages (training paradigm and the games) which suggests that some control is achievable even when the subject is trained in a different environment than they are tested within. One single subject achieved $90 \%$ online accuracy. However 6 subjects achieved over $75 \%$ accuracy in offline testing where the classifier is retrained on the first 30 blocks of the games data and tested on the remaining data. This suggests that results improve if the subject is trained within the same environment as they are tested within.

TABLE 1: Average scores for ten subjects

\begin{tabular}{l|ll}
\hline Games & Score & \% max Score \\
\hline Bowling Game & 15.4 & 77 \\
Puzzle Game & 5.3 & 66.25 \\
Shooting Game & 5 & 62.5 \\
\hline
\end{tabular}

The online session also included a final short run of each game in which the player would play the games, actively attempting to get the highest score possible. This session was conducted to assess the players using the control technique in separate game genres and also actively controlling computer games that required them to think strategically i.e., in this run the users where not instructed focus on any particular stimuli but simply follow the games mechanics. Players within these games had eight control instances thus in both the puzzle and action game the maximum score possible was 8 points, in the bowling game the player could play two rounds of bowling allowing them to achieve a maximum score of 20 points. Scores where then converted to percentages of maximum score to allow for comparison to each players accuracy (Fig. 10/Table 2).

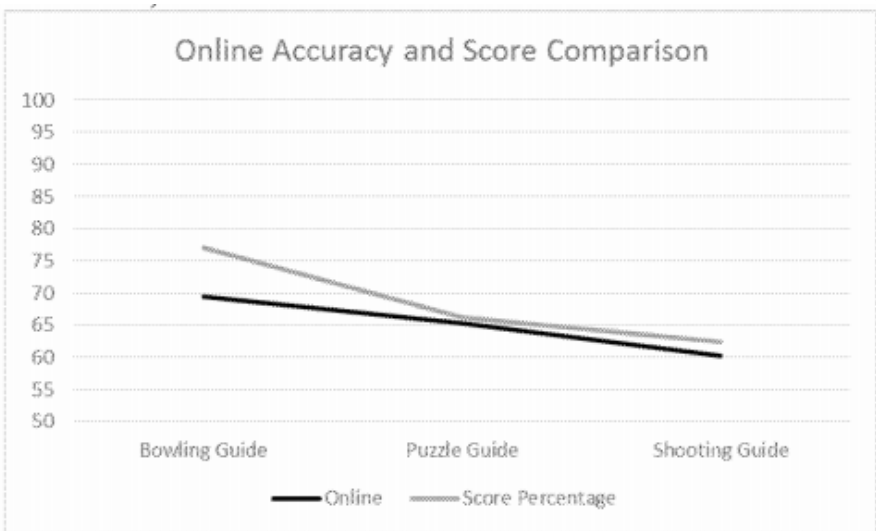

Figure 10: Comparison of Online Accuracy Percentage and percentage of max game score

The bowling game produced the highest average game score of 15.4 out of 20 . For the bowling game players achieved an average of $69.5 \%$ BCI accuracy but allowed the players to achieve $77 \%$ of the maximum score. This is because the bowling game allowed players to correct mistakes. For example, if a player chooses the bowling position incorrectly or there is a classification error, the player can then choose to bowl the ball towards the correct pins during the spin function. Techniques such as this within games allows for players to achieve high scores even if they make mistakes because of classification errors.

In both other games the player needed to choose specific correct answers to score a point. This meant that classification accuracy and percentage of maximum score were similar. In the puzzle game on average subjects achieved $65.25 \%$ BCI classification accuracy online and $66.25 \%$ of the maximum score in the games. In the shooting game subjects achieved $60.25 \%$ classification accuracy online and $62.5 \%$ of the maximum score in the games. Using a single factor ANOVA on players percentage of maximum score over the games it was found that difference between accuracies in games were not statistically significant ( $p=0.295$ ). When comparing only the bowling and shooting games $p=0.187$.

\section{DISCUSSION}

The results suggest that mVEP can be used as a control method within computer games with reasonable accuracy for a five button controller $(65 \%)$. Results also suggest that if the player was trained in the game environment that they are tested within, the player can attain a higher accuracy, $74 \%$ offline when the classifier is retrained on the game data i.e., when offline training on each level uses classifiers defined on the first 30 blocks of the data and then tested on the remaining data performance in the game is improved. This could attribute to the offline testing accuracies not being significantly different between games $(p=0.914)$. 
Online data was always conducted ten minutes (ten minutes for data analysis) after the training data was collected, results may have been degraded because of this. It should be mentioned that the time between training and testing could vary between 10 and 40 minutes depending on which game was tested first. If a game was tested 40 minutes after training the results could be degraded significantly and this may have attributed to some low accuracy results in games, which generally produce high accuracy, for example, if the bowling game was played last, some subject's results may have been considerably degraded.

In all games during the stimuli period the background around the stimuli is static, yet before and after the stimuli periods camera, character and environment changes occur. For example in the action game the environment changes every time the player is brought to a new set of targets or in the puzzle game the camera must move quickly to show the player feedback (where the ball drops to). Visual distractions before and after stimuli periods may have degraded online results as the player is not trained in levels with similar visual distractions. Different types of feedback during the games also could have caused degradation in classification. From the simple feedback in the bowling game with a ball being positioned to complex feedback with an enemy in the action game reacting to being shot. These changes in feedback may have degraded online testing results as the player is not trained in a level with comparable visual feedback.

The results from this study suggest that the genre most suited to usage with mVEP is the sports genre. With it achieving both the highest average classification accuracy and maximum score. Bowling was chosen as the sport to test in the sport genre in the game because of the paradigms slow command rate (5 Seconds per command). Sports such as bowling, golf and pool require accuracy and slow decision making, these attributes suited the mVEP based BCI paradigm. Results may differ if a sports game based around a faster paced sport such as soccer, racing or hockey, was considered because of the faster pace of decisions required and more prevalent visual distractions.

The puzzle game again was based on slow decision making, yet did not allow the player to correct mistakes. It also had a slightly more detailed environment, with tubes and walls being visible directly behind the stimuli. The action game had the worst classification accuracy and overall score. The action game had only allowed the player a relativity short time to choose the correct stimuli. This may have resulted in players concentrating on the wrong stimuli initially and thus attacking the wrong character and not scoring points. The action game also had the most graphically detailed environments with nonplayable characters, a detailed outdoor environment and with graphical enhancements such as dynamic shadows and particle effects. All of these visual distractions in the detailed environment likely degraded classification especially as the subject was not trained within such a detailed environment.

\section{LiMITATIONS}

From the results gathered it was concluded that mVEP can be used with reasonable accuracy in a variety of games. Yet by training within the game environments results could be improved. In a further study this could be explored with players being trained and then immediately tested within the same game. This would result in shorter sessions with players being trained and tested in different game genres over multiple sessions (the sessions in this study lasted up to two hours with many participants becoming fatigued in the last quarter).

Results also indicate no statistically significant difference in game genre when using the offline testing, online testing nor game score. Game score was closest to being significantly different $(p=0.295 / p=0.187)$. The lack of statistically significant results could be the fact the games do not differ significantly however it is most likely due to the limited number of subjects (10 in this study), the limited number of trials due to the testing all games in single session and the variation in performance among naïve subjects in single session. Further studies should address these issues by testing more subjects across multiple sessions where each genre of game is allocated one or more sessions. It is concluded, with the caveat of the limitation outlined above, that the game genre most suited to this paradigm is sport, giving both the highest accuracies online and the highest player score.

Another issue which may have impacted on results is the visual differences in the areas around the stimuli i.e., the buttons where overlaid over the graphics of the games. Future studies will involve testing a controller where the buttons/stimuli are contained in an area of the screen that would remain the consistent across all of the games. This could be achieved by using a Graphical user interface (GUI) that remains the same between the three game genres.

\section{CONCLUSION}

This study involved testing participants playing three different games of different genres. Subjects where trained in a separate game environment and then tested in the games. Accuracy in game control as well as how well they could play the games was investigated.

It was found that the use of mVEP as a control scheme in games is possible with reasonable online accuracy $(66 \%$ average over all games and subjects). Yet if trained within the game environment as opposed to separate training environment subjects could achieve a considerable increase in classification accuracy ( $74 \%$ offline testing accuracy). As mVEP stimuli does not visually fatigue participants it would be possible to attain this accuracy over a longer period of time than most other VEPs.

Sport was chosen as the genre that suits the paradigm as the sport chosen allowed for the required time for the subject to make a decision and concentrate on the stimuli. This study opens up the potential for further work in mVEP based BCI 
controlled computer games and testing BCI controlled games genres with different control methods. A number of key observations regarding the study design are outlined and range of recommendations for future studies are outlined.

\section{REFERENCES}

[1] D. Coyle, A. Satti, J. Stow, K. McCreadie, A. Carroll, and J. Mcelligott, "Operating a Brain Computer Interface: Able Bodied vs Physically Impaired Performance," in Proc. of the Recent Advances in Assistive Technology \& Engineering Conference, 2011.

[2] D. Coyle, J. Stow, K. McCreadie, J. McElligott, A. Carroll, "Sensorimotor Modulation Assessment and Brain-Computer Interface Training in Disorders of Consciousness," Arch. Phys. Med. Rehabil., 2015 (in press).

[3] G. Prasad, P. Herman, D. Coyle, S. McDonough, and J. Crosbie, "Applying a brain-computer interface to support motor imagery practice in people with stroke for upper limb recovery: a feasibility study.," J. Neuroeng. Rehabil., vol. 7, no. 1, p. 60, Jan. 2010.

[4] J. R. Wolpaw, N. Birbaumer, D. J. McFarland, G. Pfurtscheller, T. M. Vaughan, "Brain-computer interfaces for communication and control", Clinical Neurophysiology, vol. 113, 2002.

[5] S. Haufe, M. S Treder, M. F Gugler., M. Sagebaum, G. Curio, \& B. Blankertz, "EEG potentials predict upcoming emergency brakings during simulated driving". Journal of neural engineering, 2011.

[6] NeuroSky - Brainwave Sensors for Everybody . 2013. NeuroSky Brainwave Sensors for Everybody. [ONLINE] Available at: http://www.neurosky.com/. [Accessed 12 April 2013].

[7] Emotiv | EEG System | Electroencephalography. 2013. Emotiv | EEG System | Electroencephalography. [ONLINE] Available at: http://www.emotiv.com/. [Accessed 12 April 2013].

[8] D. Coyle, J. Garcia, A. Satti, and T. M. McGinnity, "EEG-based Continuous Control of a Game using a 3 Channel Motor Imagery BCI", IEEE Symposium Series on Computational Intelligence, 2011.

[9] D. Marshall, D Coyle, S Wilson, "Games, Gameplay and BCI: The State of the Art". Transactions on Computational Intelligence and AI in Games, 2013.

[10] F. Guo, B. Hong, X. Gao, \& S. Gao, "A brain-computer interface using motion-onset visual evoked potential". Journal of neural engineering, 5(4), 2008.

[11] B. Hong, F. Guo, T. Liu, X. Gao, \& S. Gao "N200-speller using motiononset visual response". Clinical neurophysiology : Official journal of the International Federation of Clinical Neurophysiology, 120(9). 2010.

[12] D. Coyle, J. Principe, F. Lotte, and A. Nijholt, "Guest Editorial: Brain/neuronal - Computer game interfaces and interaction," IEEE Trans. Comput. Intell. AI Games, vol. 5, no. 2, pp. 77-81, Jun. 2013

[13] D. Coyle, J. Garcia, A. Satti, and T. M. McGinnity, "EEG-based Continuous Control of a Game using a 3 Channel Motor Imagery BCI", IEEE Symposium Series on Computational Intelligence, 2011, pp. 8893,

[14] G. Müller-Putz, R. Scherer, \& G. Pfurtscheller. "Game-like training to learn single switch operated neuroprosthetic control." In BRAINPLAY 07 Brain-Computer Interfaces and Games Workshop at ACE (Advances in Computer Entertainment) 2007, 2007, pp. 41.

[15] R. Leeb, C. Keinrath, D. Friedman, C. Guger, \& C. Neuper, "Walking from thoughts: Not the muscles are crucial, but the brain waves!" Proceedings of the 8th Annual International Workshop on Presence, 2005.

[16] R. Krepki, B. Blankertz, G. Curio, \& Müller, K.-Robert. (n.d.), "The Berlin Brain-Computer Interface (BBCI) towards a new communication channel for online control of multimedia applications and computer games". Special issue on Distributed Adaptation, Representation and Processing of Multimedia Information, 2007.

[17] J. Kohlmorgen, G. Dornhege, M. Braun, B. Blankertz, K.-R. Muller, G. Curio, K. Hagemann, A. Bruns, M. Schrauf, and W. Kincses,
"Improving human performance in a real operating environment through real-time mental workload detection," Toward Brain-Computer Interfacing, 2007.

[18] B. Reuderink, A. Nijholt, and M. Poel., "Affective Pacman: A frustrating game for brain-computer interface experiments," Intelligent Technologies for Interactive Entertainment. Springer Berlin Heidelberg, 2009.

[19] U. Strehl, U. Leins, G. Goth, C. Klinger, T. Hinterberger, \& N. Birbaumer, "Self-regulation of slow cortical potentials: a new treatment for children with attention-deficit/hyperactivity disorder". Pediatrics, 118(5), 2006, pp.1530-40.

[20] H Yoon, S. W Park, Y. K Lee, and J. H Jang "Emotion Recognition of Serious Game Players Using a Simple Brain Computer Interface" ICT Convergence (ICTC), 2013 International Conference on, 2013.

[21] B. Van de Laar, H. Gurkik, D. P. Bos, M. Poal, A. Nijholt. "Experiencing BCI Controlin a Popular Computer Game". Ultra Modern Telecommunications and Control Systems and Workshops (ICUMT), 2010 International Congress on, 2010.

[22] E. C. Lalor, S. P. Kelly, C. Finucane, R. Burke, R. Smith, R. B. Reilly, \& G. McDarby, "Steady-State VEP-Based Brain-Computer Interface Control in an Immersive 3D Gaming Environment". EURASIP Journal on Advances in Signal Processing, 2005.

[23] Mühl, Christian, et al. "Bacteria Hunt: A multimodal, multiparadigm BCI game." (2010).

[24] N. Chumerin, N. V. Manyakov, A. Combaz, A. Robben, M. V. Vliet, M. M. V. Hulle, N. Manyakov, et al., "Steady state visual evoked potential based computer gaming - The Maze". The 4th International ICST Conference on Intelligent Technologies for Interactive Entertainment INTETAIN. 2011.

[25] E. C. Lalor, S. P. Kelly, C. Finucane, R. Burke, R. Smith, R.B, Reilly, \& G. McDarby, ,"Steady-State VEP-Based Brain-Computer Interface Control in an Immersive 3D Gaming Environment". EURASIP Journal on Advances in Signal Processing, 2005(19), 2005, pp. 3156-3164.

[26] A. Finke, A. Lenhardt, H. Ritter, "The MindGame: A P300-based braincomputer interface game", Neural Networks, Volume 22, Issue 9, November 2009, 2009, pp. 1329-1333.

[27] D. Marshall, D. Coyle, \& S. Wilson (2013, June). Motion-onset visual evoked potentials for gaming: A pilot study. In Signals and Systems Conference (ISSC 2013), 24th IET Irish (pp. 1-7). IET.

[28] Unity - Game Engine. 2013. Unity - Game Engine. [ONLINE] Available at: http://unity3d.com/. [Accessed 11 April 2013].

[29] MATLAB and Simulink for Technical Computing - MathWorks United Kingdom. 2013. MATLAB and Simulink for Technical Computing MathWorks United Kingdom. [ONLINE] Available at: http://www.mathworks.co.uk/. [Accessed 15 April 2013].

[30] "International 10-20 System." [Online]. Available http://en.wikipedia.org/wiki/10-20_system_(EEG). [Accessed: 29-May2014].

[31] BioSig. 2015. BioSig. [ONLINE] Available at: http://biosig.sourceforge.net/. [Accessed 12 January 2015].

[32] "g.tec - g.BSamp Biosignal Amplifier," 2014. [Online]. Available: http://www.gtec.at/Products/Hardware-and-Accessories/g.BSampSpecs-Features. [Accessed: 29-May-2014].

[33] "g.Tec - g.GAMMAbox," 2014. [Online]. Available: http://www.gtec.at/Products/Electrodes-and-Sensors/g.GAMMAsysSpecs-Features. [Accessed: 29-May-2014].

[34] LIBSVM -- A Library for Support Vector Machines. 2015. LIBSVM -A Library for Support Vector Machines. [ONLINE] Available at: http://www.csie.ntu.edu.tw/ cjlin/libsvm/. [Accessed 12 January 2015].

[35] H. J. Heinze, et al 1994 "Combined spatial and temporal imaging of brain activity during visual selective attention in humans" Nature 372 , 1994.

[36] A. M. Hollants-Gilhuijs, J. C. DeMunck, Z. Kubova, E. van Royen and E. Spekreijse, "The development of hemispheric asymmetry in human motion VEPs", Vision Research 40, 2000. 